\title{
DOUBLE FAULT DISTINGUISHABILITY IN LINEAR SYSTEMS
}

\author{
JAN MACIEJ KOŚCIELNY, ZOFIA M. ŁABĘDA-GRUDZIAK \\ Institute of Automatic Control and Robotics \\ Warsaw University of Technology, ul. św. Andrzeja Boboli 8, 02-525 Warsaw, Poland \\ e-mail: \{jmk, z.labeda\} @mchtr.pw.edu.pl
}

\begin{abstract}
This paper develops a new approach to double fault isolation in linear systems with the aid of directional residuals. The method of residual generation for computational as well as internal forms is applied. Isolation of double faults is based on the investigation of the coplanarity of the residual vector with the planes defined by the individual pairs of directional fault vectors. Additionally, the method of designing secondary residuals, which are structured and directional, is proposed. These transformations allow achieving various isolation properties. It is shown that double fault distinguishability can be improved by decomposing the observed residual vector along the response directions. The described methods are illustrated with a simple computational example.
\end{abstract}

Keywords: faults isolation, double fault, fault distinguishability, directional and structured residuals, secondary residuals, linear systems.

\section{Introduction}

Fault distinguishability is one of the most important issues defining the quality of diagnostics of technical systems. Higher fault distinguishability results in a more accurate diagnosis. Therefore, an improvement of fault distinguishability leads to an increase in the overall diagnosing accuracy. More accurate diagnostics means lower power subsets of faults.

The paper deals with the problem of double fault isolation. On-line diagnostics of industrial processes is mostly performed with the assumption of single faults (Gertler, 1998; Chen and Patton, 1999; Patton et al., 2000; Isermann, 2006; Korbicz et al., 2004). Such an assumption causes a considerable simplification of the algorithm of fault isolation. However, the problem of admissibility of such assumption seems to be disputable. In large-scale technological installations, due to the plant scale and reliability factors, faults have to be considered unavoidable. They may appear with differentiated frequency as a single or multiple faults. A multiple fault means simultaneous occurrences of any possible combination of single faults. The most difficult for isolation are particularly the faults that appear simultaneously. If $n$ is the number of possible faults, then there are $2^{n}$ possible system states with multiple faults. This shows the scale of the problem. Therefore it is necessary to take into consideration not only single faults, but also multiple ones. First of all, we should analyze the possibility of double fault occurrence.

One can distinguish two main groups of model-based approaches to multiple faults diagnosis. The first one is based on the knowledge of nonlinear or linear models of the system, but without including the influence of faults on the system output. Well-known examples are state and output equations which can generally be written as

$$
\begin{aligned}
\dot{\mathbf{x}}(t) & =\phi[\mathbf{x}(t), \mathbf{u}(t)], \\
\mathbf{y}(t) & =\psi[\mathbf{x}(t), \mathbf{u}(t)],
\end{aligned}
$$

where $\mathbf{x}, \mathbf{u}$ and $\mathbf{y}$ are the state, input, and output vectors, respectively, while $\phi$ and $\psi$ are nonlinear continuous functions with certain smoothness properties. The relationship that exists between faults and residual values generated by the models of the system usually takes the form of a binary relation and is determined by expert knowledge or learning methods.

In this group of works it is necessary to mention the approaches coming from the automatic control area and the FDI community compared with the ones based on Artificial Intelligence (AI) techniques and developed by the DX community (a series of conferences devoted to automated diagnosis methods emerging from AI). Artificial intelligence-based methods called model-based diagnosis as well as methods based on the theory of 
Reiter (1987) allow isolating single and multiple faults (de Kleer and Williams, 1987; Górny, 2001; Hamscher et al., 1992; Hwee, 1991; de Kleer and Kurien, 2003; Daigle et al., 2006; Ligęza and Kościelny, 2008). The diagnoses are generated as minimal hitting sets of all the minimal conflict sets. These approaches take advantage by allowing for fault compensation effects. But they found applications only for diagnostics of relatively simple systems.

This group of methods may also include an approach to qualitative isolation of multiple faults described by Daigle et al. (2006). It is based on the TRANSCEND framework (Mosterman and Biswas, 1999; Manders et al., 2000), which employs a qualitative approach for analysis of fault transient behavior. The diagnosis model is used to generate fault signatures, which represent the magnitude and higher order effects of faults on the measurements.

In the FDI area, one of the first approaches applied to isolation of multiple faults of instruments and actuators was carried out with the application of a bank of observers for residual generation and classic logic for decision making procedures about faults (Frank, 1987; Clark, 1989). The common FDI approach is fault detection and fault isolation based on elementary component models (analytical, fuzzy or neural) of physical systems and a binary diagnostic matrix, which presents the relation existing between bi-state diagnostic signals and faults (Kościelny, 1995; 2001; Kościelny et al., 2012; Staroswiecki et al., 2000). Signatures for states with multiple faults are created based on the binary diagnostics matrix (Gertler, 1998; Staroswiecki et al., 2000; Korbicz et al., 2004). A different approach exploits the assumed availability of data from process faulty states. Sorsa and Koivo (1993), Watanabe and Hirota (1991) as well as Watanabe and Hou (1992) applied neural networks to model relations between faults and fault symptoms in the diagnostics of industrial processes. But it is clear that acquisition of such data is practically impossible.

Models of the second group include the influence of faults $\mathbf{f}$, and sometimes noise. Faults are treated as peculiar inputs of an object. The description of a dynamic system with respect to faults can be represented by the following equations:

$$
\begin{aligned}
\dot{\mathbf{x}}(t) & =\phi[\mathbf{x}(t), \mathbf{u}(t), \mathbf{f}(t)], \\
\mathbf{y}(t) & =\psi[\mathbf{x}(t), \mathbf{u}(t), \mathbf{f}(t)] .
\end{aligned}
$$

In this case, residual values explicitly depend on faults. Basically, there are two fundamental frameworks to create a residual set to enable fault isolation. One approach is to design a set of structured residual signals. Each residual is designed to be sensitive to a certain group of faults, while being insensitive to others. Another way of isolation is to design a directional residual vector, which means forcing the residual vector to lie in a fixed and fault-specific direction in the residual space with respect to each fault (Gertler, 1998; Korbicz et al., 2004). If the diagnosis is carried out with linear input-output type models, not only the calculation form of residuals must be determined, but also the inner form, which defines the dependence of residuals on faults (Gertler, 1998; Chen and Patton, 1999; Patton et al., 2000; Korbicz et al., 2004).

Methods in this group create potentially more possibilities of multiple faults isolation. This is due to a much higher level of knowledge about relation between residual values and faults. However, in the case of structured residuals (Gertler, 1998), the fault isolation rule consists in concluding based on the binary diagnostics matrix mentioned previously. This means that distinguishability of faults does not improve.

Knowledge about the internal form of residuals enables fault sensitivity evaluation with the use of fault decoupling along with disturbances (Gertler, 1998; Frank, 1991). The objective is to achieve a unitary matrix, which contains ones on its diagonal. Then the number of residuals equals that of faults. A characteristics feature of the matrix is that each diagnostic signal detects one fault only, different from those detected by the other diagnostic signals. Such a diagnostic matrix is the optimal solution. It ensures a strongly isolating distinguishability of faults. Then, the problem of multiple faults can be solved.

Khémiri et al. (2011) presented a new recursive optimal filter structure. The fault affects both the state and output equations, whereas unknown disturbances only affect the state system equation, without any prior information about their dynamical evolution. The resulting filter was applied to solve simultaneous actuator and sensor fault estimation. Hashtrudi and Massoumnia (1999) also analyzed simultaneous actuator and sensor faults. A result of the theorem is that when actuator and sensor failure modes are scalar, the EFPRG (Extended Fundamental Problem in Residual Generation) is generically solvable if and only if the number of actuator failure modes is less than or equal to the dimension of the state space of the system and the total number of actuator and sensor failure modes is less than or equal to the number of system outputs.

However, a diagonal diagnostic matrix can be achieved only if the number of faults is less than or equal to the number of system outputs (Gertler, 1998). In fact, the number of system outputs is equal to that of instrument faults. If we take into consideration all the faults, i.e., instrument, actuator and component faults, then the number of faults is always greater than that of system outputs. Thus, this approach is not useful in industrial practice.

Other techniques were also proposed for multiple fault isolation. The method of detection filters (Chen and Speyer, 1999) and application of decoupled Kalman filters (Adam-Medina et al., 2003) as well as decoupled 
unknown input observers (Ding, 2008) potentially give substantial possibilities of isolation of multiple faults. De-Persis and Isidori (2001) studied the problem of fault detection and isolation for nonlinear systems. The extension to the case of multiple concurrent faults was briefly discussed. Mattone and de Luca (2006) described a geometric approach to nonlinear robotic systems. The proposed approach uses the hybrid structure of the diagnostic system, cascading nonlinear residual generators with combinatorial isolation logics for detection and isolation multiple fault. However, this method differs from the one presented in this paper. Verde et al. (2001) show how fuzzy directional residual evaluation can be used to isolate multileaks in pipelines in a fuzzy framework.

In this paper we present the possibilities of double fault isolation for a linear system of equations or linearized in a neighbourhood of an operating point, assuming that parity equations are known in computational forms as well as internal forms (Gertler, 1998; Kościelny, 2001; Korbicz et al., 2004), i.e., relationships are known not only between input and output signals of the diagnosed object but also between faults and output signals.

The contribution in the present paper is an extension of the residual vector generation method (Gertler, 1998) to increase double fault distinguishability. The presented approach is based on directional residuals and investigation of the coplanarity of the residual vector with the planes defined by individual pairs of directional fault vectors. This method allows distinguishing many types of double faults, which are not distinguished on the basis of binary signatures in the structured approach and AI methods. This paper is a continuation of our previous work (Kościelny and Łabęda, 2007).

This paper is organized as follows. In Section 2, we list out the assumptions under which the analysis of the problems of double faults distinguishability is carried out. The dynamic consistence equations calculated from transfer function models, which take the effect of faults into account, are also presented in Section 2. For the design of directional residuals, the calculation and the internal form of primary residuals are derived. In Section 3, we introduce definitions and notation required to describe the graphical interpretation of double faults in the parity space. Section 4 describes the principles of formulating the hypotheses about occurrence of double faults. In Section 5, we derive double fault distinguishability conditions. Section 6 deals with the design of structured residuals. The technique of residual enhancement by design of a secondary residual, which improves double fault isolability, is developed. The approach is then illustrated on a simple, computational example in Section 7. The paper concludes with Section 8.

\section{Description of linear diagnosed objects taking into consideration the influence of faults}

The analysis of problems of distinguishability of multiple faults is carried out under following assumptions:

1. All single faults in the system are distinguished on the basis of any fault isolation method, such as structured or directional residuals. If the condition is not satisfied, then the reasonableness of multiple faults distinguishability, in particular double faults, is very limited. Only if the distinguishability of single faults is achieved it is purposeful to analyze the distinguishability of double faults.

2. The residual response to double faults can be derived from a single fault response. This assumption should hold in most real applications.

3. The system is linear or can be made, with a sufficient accuracy approximate linear description, valid in the surroundings of a working point.

4. The influence of faults on outputs of the system as well as residuals can be described, with sufficient accuracy, by the linear equations (4). Note that this is a strong assumption, limiting the applicability of the proposed method since the nature of faults is strongly nonlinear.

5. No transfer functions describing the system have an integral term. This restriction applies mainly to the method of directional residuals. It usually holds in practice.

6. The fault set $F$ size is greater than sets of inputs $U$ and outputs $Y$ of the system, i.e., $|F|>|U|,|Y|$.

7. The influence of disturbances and noise is omitted.

In order to isolate faults, it is necessary to have knowledge of the relationships between faults and values of diagnostic signals. This relationship can be defined as a result of object modeling taking into consideration influence of faults (2). Real systems are usually nonlinear, but for simplification of the mathematical description their linearization is conducted. It allows us to formulate an approximate linear description, valid in the surroundings of an operating point on the static characteristic (this point, most often, corresponds to nominal or average working conditions). At every operating point the function $\phi$ is linearized. A similar Taylor expansion can be made for the function $\psi$. An approximation of (2) with local linear models is

$$
\begin{aligned}
\dot{\mathbf{x}}(t) & =A \mathbf{x}(t)+B \mathbf{u}(t)+E \mathbf{f}(t), \\
\mathbf{y}(t) & =C \mathbf{x}(t)+D \mathbf{u}(t)+F \mathbf{f}(t),
\end{aligned}
$$


where $\mathbf{x}$ is a state vector, which characterizes the internal state of the system at any instant $t, \mathbf{y}$ is an output vector, $\mathbf{u}$ is an input vector, $\mathbf{f}$ is a fault vector, matrices $A, B, C, D$, $E$ and $F$ are the parameters of the state space realization.

A commonly used form of the description of a linear process is a transfer function model containing the set of equations defining the relationship between process outputs, inputs and faults (Geltler and Singer, 1990; Gertler, 1998):

$$
\mathbf{y}(s)=\mathbf{G}(s) \mathbf{u}(s)+\mathbf{G}^{F}(s) \mathbf{f}(s) .
$$

The transfer functions $\mathbf{G}(s)$ and $\mathbf{G}^{F}(s)$ are all assumed to be proper and of suitable dimensions. Particular equations have the following form:

$$
\begin{aligned}
y_{j}(s)= & G_{j 1}(s) u_{1}(s)+\cdots+G_{j P} u_{P}(s) \\
& +G_{j 1}^{F}(s) f_{1}(s)+\cdots+G_{j K}^{F} f_{K}(s),
\end{aligned}
$$

where $G_{j p}(s)$ for $j=1, \ldots, J$ and $p=1, \ldots, P$ is the transfer function between the $j$-th output and the $p$-th input:

$$
G_{j p}(s)=\frac{y_{j}(s)}{u_{p}(s)},
$$

while $G_{j k}^{F}(s)$ for $j=1, \ldots, J$ and $k=1, \ldots, K$ is the transfer function between the $j$-th output and the $k$-th fault:

$$
G_{j k}^{F}(s)=\frac{y_{j}(s)}{f_{k}(s)} .
$$

In the case of a fault free state, the dependence $\mathbf{y}(s)-\mathbf{G}(s) \mathbf{u}(s)=\mathbf{G}^{F}(s) \mathbf{f}(s)=\mathbf{0}$ is fulfilled. Therefore, symptoms of faults are non-zero residuals, calculated from the following primary residual form:

$$
\mathbf{r}(s)=\mathbf{y}(s)-\mathbf{G}(s) \mathbf{u}(s),
$$

called the computational residual form. The general relation between residual and faults defines the so-called internal residual form:

$$
\mathbf{r}(s)=\mathbf{G}^{F}(s) \mathbf{f}(s) .
$$

The internal form of the $j$-th residual is then

$$
r_{j}(s)=G_{j 1}^{F}(s) f_{1}(s)+\cdots+G_{j K}^{F}(s) f_{K}(s) .
$$

This relation can be represented, for all the residuals, in the form shown in Table 1. The dynamics of the $j$-th residual under the influence of the $k$-th fault are described by the transfer function $G_{j k}^{F}(s)$. Thus, having the description of fault influence on the residuals in the internal form (10), it is possible to define the sensitivity of residuals to individual faults, not only qualitatively, but also quantitatively. The set of transfer functions from the column of Table 1 corresponding to particular fault defines its signature.

Table 1. Internal form of residuals.
\begin{tabular}{|c|c|c|c|c|c|}
\hline & $f_{1}$ & $\cdots$ & $f_{k}$ & $\cdots$ & $f_{K}$ \\
\hline \hline$r_{1}$ & $G_{11}^{F}$ & & $G_{1 k}^{F}$ & & $G_{1 K}^{F}$ \\
\hline$\ldots$ & & & & & \\
\hline$r_{j}$ & $G_{j 1}^{F}$ & & $G_{j k}^{F}$ & & $G_{j K}^{F}$ \\
\hline$\ldots$ & & & & & \\
\hline$r_{J}$ & $G_{J 1}^{F}$ & & $G_{J k}^{F}$ & & $G_{J K}^{F}$ \\
\hline
\end{tabular}

According to Assumption 5 the individual transfer function $G_{j k}^{F}$ in Eqn. (10) has no integral term. In this case, the values of the transfer function in the column corresponding to a particular fault define the gain and dynamics of residuals, which are sensitive to this fault. In steady state, i.e., when transient processes have already decayed (theoretically, the time of the proceeding of system to steady state is infinitely long, therefore for $t \rightarrow \infty)$, it is possible to calculate, on the basis of the theorem about the final value, the gain $c_{j k}$ for individual pairs of the $k$-th fault and the $j$-th residual. Therefore, for $f_{k}(t)=\mathbf{1}(t)$ with the transfer function $f_{k}(s)=1 / s$, $k=1, \ldots, K$, we get

$$
\lim _{t \rightarrow \infty} r_{j}(t)=\lim _{s \rightarrow 0} s r_{j}(s)=\lim _{s \rightarrow 0} s G_{j k}^{F} \frac{1}{s}=c_{j k} .
$$

The set of linear equations is given in Table 2 Since $J<K$ (Assumption 6), the system is unsolvable

Table 2. Directional signatures.

\begin{tabular}{|c|c|c|c|c|c|}
\hline & $f_{1}$ & $\ldots$ & $f_{k}$ & $\ldots$ & $f_{K}$ \\
\hline \hline$r_{1}$ & $c_{11}$ & & $c_{1 k}$ & & $c_{1 K}$ \\
\hline$\ldots$ & & & & & \\
\hline$r_{j}$ & $c_{j 1}$ & & $c_{j k}$ & & $c_{j K}$ \\
\hline$\ldots$ & & & & & \\
\hline$r_{J}$ & $c_{J 1}$ & & $c_{J k}$ & & $c_{J K}$ \\
\hline
\end{tabular}

in a general case. If $f(t)$ is of binary nature $f_{k}(1)=$ $1(t)$, then the solution is in $\{0,1\}^{K}$ space. We can solve this algebraic equation by integer programming. The solution will not be unique, each corresponding to a single diagnostic hypothesis. However, there is no need to assume $f_{k}(1)=1(t)$. We therefore propose the use of directional residuals.

The vector of steady state residual gains, in response to a particular fault $f_{k}$, defines some direction $\mathbf{w}_{k}=$ $\left[c_{1 k}, \ldots, c_{j k}, \ldots, c_{J k}\right]$ in the residual space (called the parity space) and lies in a fixed and that fault-specific space at all times, including transients. A fault is then isolated by determining the fault signature direction that is the closest to the generated residual vector. It is the base of the directional residual method (Gertler, 1998; Patton et al., 2000; Chen and Patton, 1999).

So far, directional residuals have been analysed mainly in the context of single fault isolation. In this paper 
an attempt to improve the performance of double fault distinguishability is made.

\section{Graphical interpretation of double faults in the parity space}

Let us assume that the residual vector is confined to a fault specific direction $\mathbf{w}_{k}=\left[c_{1 k}, \ldots, c_{J k}\right]$. The isolation approach presented in this paper uses a three-dimensional parity space for a system of residuals $\mathbf{r}=\left[r_{1}, r_{2}, r_{3}\right]$ and a set of binary detection (Boolean) tests to compose the observed fault signature. However, the use of binary codification of the residual produces a lack of information that can lead to wrong diagnosis. This happens in some double faults that are indistinguishable because they present the same theoretical binary fault signature. Our approach is based on the analysis of such cases of double faults. The three-dimensional space of residuals is sufficient for our purposes. The residuals must be properly selected from the whole set of residuals. We mainly choose the residuals which are sensitive to the largest number of faults form the analyzed pairs of faults.

The directional vectors $\mathbf{w}_{k}=\left[c_{1 k}, c_{2 k}, c_{3 k}\right]$ and $\mathbf{w}_{m}=\left[c_{1 m}, c_{2 m}, c_{3 m}\right]$ for optional pairs of faults $f_{k}$ and $f_{m}$ determine the plane $\mathbf{H}_{k m}$ in the parity space. It is possible to calculate the equation of the plane by defining a perpendicular vector to this plane and taking into consideration that it includes the initial point of the coordinate system.

Assume that $\mathbf{w}_{k}$ and $\mathbf{w}_{m}$ are linear independent. Then $\mathbf{w}_{k m}=\mathbf{w}_{k} \times \mathbf{w}_{m}$ is a non-zero vector perpendicular to both vectors, $\mathbf{w}_{k}$ and $\mathbf{w}_{m}$, and perpendicular to the plane $\mathbf{H}_{k m}$ that contains them. The definition of the vector product can be represented by the determinant of a formal matrix:

$$
\begin{aligned}
\mathbf{w}_{k m} & =\mathbf{w}_{k} \times \mathbf{w}_{m}=\left|\begin{array}{ccc}
\mathbf{i} & \mathbf{j} & \mathbf{k} \\
c_{1 k} & c_{2 k} & c_{3 k} \\
c_{1 m} & c_{2 m} & c_{3 m}
\end{array}\right| \\
& =a_{1 k m} \mathbf{i}+a_{2 k m} \mathbf{j}+a_{3 k m} \mathbf{k},
\end{aligned}
$$

where the unit vectors $\mathbf{i}, \mathbf{j}, \mathbf{k}$ are the versors of the coordinate system $r_{1}, r_{2}, r_{3}$, and the vectors of the standard basis in three dimensions. The three scalar components of the resulting vector $\mathbf{w}_{k m}$ are

$$
\begin{aligned}
& a_{1 k m}=c_{2 k} c_{3 m}-c_{3 k} c_{2 m}, \\
& a_{2 k m}=c_{3 k} c_{1 m}-c_{1 k} c_{3 m}, \\
& a_{3 k m}=c_{1 k} c_{2 m}-c_{2 k} c_{1 m} .
\end{aligned}
$$

The equation of the plane $\mathbf{H}_{k m}$ passing through the coordinate origin and perpendicular to $\mathbf{w}_{k m}$ is in the following form:

$$
a_{1 k m} r_{1}+a_{2 k m} r_{2}+a_{3 k m} r_{3}=0 .
$$

The sample plane $\mathbf{H}_{k m}$ in the parity space is represented by the grey colour on the diagram of Fig. 1

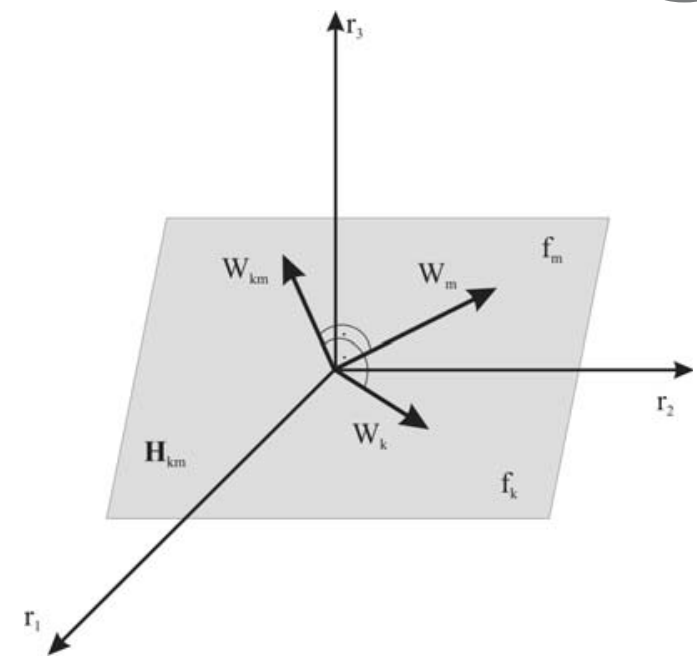

Fig. 1. Diagram of the plane $\mathbf{H}_{k m}$ in three-dimensional coordinate systems $r_{1}, r_{2}, r_{3}$

\section{Principles of formulating hypotheses about occurrence of double faults}

In a steady state, assuming the appearance of double fault $f_{k}, f_{m}$ and no other faults, the vector of residuals $\mathbf{r}$ should be confined to the fault specific plain $\mathbf{H}_{k m}$. For steady state conditions, if the current vector is coplanar with the plane $\mathbf{H}_{k m}$ of proper pairs of faults, i.e., if the scalar triple product is equal to zero,

$$
\left(\mathbf{w}_{k} \times \mathbf{w}_{m}\right) \cdot \mathbf{r}=a_{1 k m} r_{1}+a_{2 k m} r_{2}+a_{3 k m} r_{3}=0,
$$

then the occurrence of the pair of faults $f_{k}$ and $f_{m}$ can be hypothesized. Obviously, other combinations of a pair of faults can define the same plane (e.g., it is enough to have Table 2 with identical columns). Then, these faults are indistinguishable. Therefore, the hypothesis statement about occurrence of double faults amounts to determining to which pre-defined double fault specific plane the observed residual vector lies the closest. This directly implies the double fault isolation method.

In fact, the actual residual vector may not coincide precisely with the planes of proper pairs of faults. This may be due to the presence of noise, disturbances and modeling errors. Thus, the coplanarity condition (15) is not satisfied. The measure of the consistency of actual residual vector and the plane of double fault $f_{k}, f_{m}$ was determined using the angle between the vector $\mathbf{r}$ and the plane $\mathbf{H}_{k m}$.

Let $\varphi$ be the angle between $\mathbf{r}$ and the normal $\mathbf{w}_{k m}$ to the plane $\mathbf{H}_{k m}$. Then the cosine of this angle can be calculated as follows:

$$
\cos \varphi=\frac{\mathbf{r} \cdot \mathbf{w}_{k m}}{\|\mathbf{r}\|\left\|\mathbf{w}_{k m}\right\|}
$$

This formula involves the dot product and the magnitudes of two vectors. Therefore, the size of the angle $\alpha_{k m}$ 
between residual vector $\mathbf{r}$ and plane $\mathbf{H}_{k m}$ of double fault $f_{k}, f_{m}$ is given by

$$
\alpha_{k m}=90^{\circ}-\arccos \varphi .
$$

As the value of $\alpha_{k m}$ gets closer to zero, the hypothesis about the occurrence of a double fault $f_{k}, f_{m}$ is more certain. The isolability of double faults can be achieved by comparison of the size of the angles $\alpha_{k m}$ in response to the different pairs of faults $f_{k}$ and $f_{m}$. It should be stressed that various combinations of double faults may lead to the same plane, so any other pair of faults may lead to the plane with a similar or the same $\alpha_{k m}$.

\section{Double fault distinguishability}

The possibility of fault distinguishability is vital in the design of a diagnostics system. In the case of directional residuals, fault distinguishability depends on the existing faults-specific directions. The diagnostic system is required to decide whether it is possible to achieve the distinguishability for a given pair of faults from other faults and other pairs of faults. Assuming the steady state, the following can be hypothesized:

- A fault $f_{n}$ will be distinguishable from a double fault $f_{k}, f_{m}$, where $n \neq k, m$ and $k, m, n \in\{1 \ldots, K\}$, on the basis of optional three residuals, when the directional vector of this fault does not lie on the plane determined by fault specific directions of $f_{k}$ and $f_{m}$, i.e., these three vectors are not coplanar: $\left(\mathbf{w}_{k} \times \mathbf{w}_{m}\right) \cdot \mathbf{w}_{n} \neq 0$.

- Two pairs of faults $f_{k}$ and $f_{m}$, and $f_{l}$ and $f_{n}$ for $k, l, m, n \in\{1 \ldots, K\}$ are distinguishable on the basis of three optional residuals, when the corresponding planes $\mathbf{H}_{k m}$ and $\mathbf{H}_{l n}$ do not coincide and the current vector of residuals $\mathbf{r}$ does not coincide with the intersection of these two planes.

- If three or more fault specific directions are coplanar (scalar triple product of the given three vectors is equal to zero), then there is no possibility to distinguish double faults in the set of faults under consideration.

\section{Design for structured residuals}

Designing the residual set with a certain structure is one of the ways of enhancing its fault isolation capabilities. Structured residuals are designed in such a way that each residual responds to a different subset of faults and is insensitive to others (Geltler and Singer, 1990). The sensitivity is written down in the binary diagnostic matrix which is defined on the Cartesian product of the binary set of residuals and faults. If the residual is sensitive to a fault, an appropriate element of the matrix becomes equal to one, otherwise it is equal to zero. In order to improve the distinguishability of double faults and achieve robustness issues, it is possible to design additional secondary residuals, which are structured and directional (Gertler, 1998). They should ensure the possibility of the distinguishability of double faults that are indistinguishable on the basis of primary residuals. The aim is to decompose the observed residual vector along the response directions.

The secondary residuals $\mathbf{r}^{*}$ can be obtained by multiplying primary residuals $\mathbf{r}$ by a linear transformation,

$$
\mathbf{r}^{*}(s)=\mathbf{V}(s) \mathbf{r}(s)=\mathbf{V}(s) \mathbf{G}^{F}(s) \mathbf{f}(s),
$$

where $\mathbf{V}$ is a transfer function matrix. It should be noted that in fact this is a post-filtering primary residual method, where $\mathbf{V}$ is a filter transfer matrix. Designing the secondary residual $\mathbf{r}^{*}$ set amounts to selecting the transforming matrix $\mathbf{V}$ so that the residuals $\mathbf{r}^{*}$ possess certain desired properties. The residuals transformation should be selected in such a way that the planes $\mathbf{H}_{k m}$, in response to double faults $f_{k}, f_{m}$, have different location in the parity space.

If we consider a three-dimensional parity space, it is advantageous to choose the transforming matrix $\mathbf{V}(s)$ so that the response directions $\widetilde{\mathbf{w}}_{k}, \widetilde{\mathbf{w}}_{m}, \widetilde{\mathbf{w}}_{n}$ of single faults $f_{k}, f_{m}$ and $f_{n}$ constitute an orthogonal set. Thus, the observed residual vectors will occur in double faults $f_{k}, f_{m}$, and $f_{m}, f_{n}$ and $f_{k}, f_{n}$ specific planes $\mathbf{H}_{k m}, \mathbf{H}_{m n}$ and $\mathbf{H}_{k n}$, which are perpendicular to each other. With orthogonal directions, double faults can be isolated, at least in principle. As will be seen, the number of faults for which such properties can be achieved is limited to that of plant outputs. Perpendicular response directions for $K$ faults require a residual space of dimension $K$ (Gertler, 1998).

Figure 2 presents sample response directions and orthogonal set of surfaces in a three-dimensional parity space.

The two vectors $\widetilde{\mathbf{w}}_{k}=\left[b_{1 k}, b_{2 k}, b_{3 k}\right]$ and $\widetilde{\mathbf{w}}_{m}=$ $\left[b_{1 m}, b_{2 m}, b_{3 m}\right]$ are perpendicular if their dot-product equals zero,

$$
\widetilde{\mathbf{w}}_{k} \cdot \widetilde{\mathbf{w}}_{m}=b_{1 k} b_{1 m}+b_{2 k} b_{2 m}+b_{3 k} b_{3 m}=0 .
$$

With the above dependence, we can generate a set of residual vectors in which all vectors are perpendicular to one another. The task is to define a transfer function matrix $\mathbf{V}$ so that the secondary residuals $\mathbf{r}^{*}$ are such that the appearance of the $k$-th fault causes the residual vector $\mathbf{r}^{*}$ to change in the direction $\widetilde{\mathbf{w}}_{k}$.

It is essential that the dynamics of the directional response remain the same for each element of the residual vector, otherwise the directional property would not be maintained during fault transients. Thus, a way of enhancing the diagnostics utility of residuals is to make 


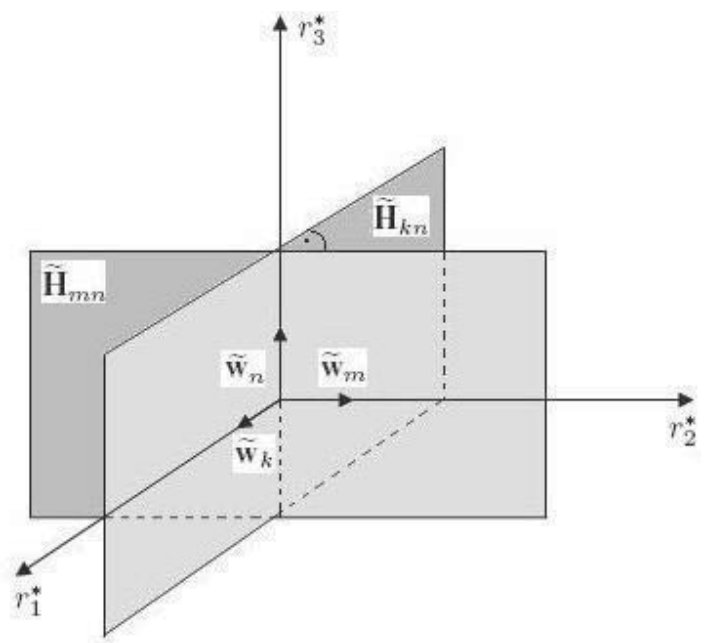

Fig. 2. Graph of sample surfaces $\widetilde{\mathbf{H}}_{k n} \perp \widetilde{\mathbf{H}}_{m n}$ in threedimensional coordinate systems $r_{1}^{*}, r_{2}^{*}, r_{3}^{*}$.

them lie in a fixed and fault specific direction in the residual space. The response of the residual $\mathbf{r}^{*}(s)$ to the faults $f_{k}(s)$ is as follows:

$$
\mathbf{r}^{*}\left(s / f_{k}\right)=\widetilde{\mathbf{w}}_{k} D_{k}(s) f_{k}(s)
$$

where the vector $\widetilde{\mathbf{w}}_{k}$ is the direction of the $k$-th fault response and the scalar transfer function $D_{k}(s)$ (polynomial or rational function) is its dynamic. For a set of $K$ faults we should determine $K$ directions of the $K$ fault responses,

$$
\mathbf{W}=\left[\widetilde{\mathbf{w}}_{1}, \widetilde{\mathbf{w}}_{2}, \ldots, \widetilde{\mathbf{w}}_{K}\right]
$$

and the diagonal matrix $\mathbf{D}(s)$,

$$
\mathbf{D}(s)=\left[\begin{array}{cccc}
D_{1}(s) & 0 & \ldots & 0 \\
0 & D_{2}(s) & \ldots & 0 \\
\vdots & \vdots & \ddots & \vdots \\
0 & 0 & \ldots & D_{K}(s)
\end{array}\right]
$$

Therefore, the fault response specifications 20 can be combined, for $k=1, \ldots, K$, as

$$
\mathbf{r}^{*}=\mathbf{W D}(s) \mathbf{f}(s) \text {. }
$$

Equations (18) and 23) imply that

$$
\mathbf{W D}(s)=\mathbf{V}(s) \mathbf{G}^{F}(s) .
$$

The size of matrices are $\mathbf{G}_{J \times K}^{F}, \mathbf{W}_{N \times K}, \mathbf{D}_{K \times K}$ and $\mathbf{V}_{N \times J}$. We wish to design the transformation so that all fault responses have independent directions. For such directions, the fault transfer matrix $\mathbf{G}^{F}$ must have full column rank. This implies that the matrix $\mathbf{G}^{F}$ has an inverse $\left[\mathbf{G}^{F}\right]^{-1}$. It should be noted that the number of faults for which independent directional responses can be designed is limited to that of outputs, and also the number of transformed residuals may not be less than that of faults. The transformation $\mathbf{V}(s)$ can be obtained from Eqn. (24) as

$$
\mathbf{V}(s)=\mathbf{W D}(s)\left[\mathbf{G}^{F}(s)\right]^{-1} .
$$

An important special case, which will be referred to as the basic set of residuals (Gertler, 1998), arises when

$$
\begin{aligned}
& J=K, \\
& \mathbf{W}=\mathbf{I}, \\
& D_{1}(s)=D_{2}(s)=\ldots=D_{K}(s)=\widetilde{D}(s),
\end{aligned}
$$

where I is the identity matrix. The basis set is very convenient for double fault isolation, because all possible double faults have distinct signatures. Then the matrix $\widetilde{\mathbf{D}}(s)$ becomes

$$
\widetilde{\mathbf{D}}(s)=\left[\begin{array}{cccc}
\widetilde{D}(s) & 0 & \ldots & 0 \\
0 & \widetilde{D}(s) & \ldots & 0 \\
\vdots & \vdots & \ddots & \vdots \\
0 & 0 & \ldots & \widetilde{D}(s)
\end{array}\right]
$$

In this case Eqn. (24) can be rephrased as

$$
\widetilde{\mathbf{D}}(s)=\mathbf{V}(s) \mathbf{G}^{F}(s) .
$$

The above expressions finally yield the following specification of $\mathbf{V}(s)$ :

$$
\mathbf{V}(s)=\widetilde{\mathbf{D}}(s)\left[\mathbf{G}^{F}(s)\right]^{-1} .
$$

With a general form of matrix $\mathbf{V}(s)$ 25), the internal form of residuals $\mathbf{r}^{*}$ is obtained as

$$
\begin{aligned}
\mathbf{r}^{*}(s) & =\mathbf{V}(s) \mathbf{G}^{F}(s) \mathbf{f}(s) \\
& =\mathbf{W D}(s)\left[\mathbf{G}^{F}(s)\right]^{-1} \mathbf{G}^{F}(s) \mathbf{f}(s) \\
& =\mathbf{W D}(s) \mathbf{I f}(s)=\mathbf{W D}(s) \mathbf{f}(s) .
\end{aligned}
$$

\section{Example}

Consider the following system with three outputs $y_{j}, j=$ $1,2,3$, depending on input signals $u_{p}, p=1, \ldots, 4$, and from appearing faults $f_{k}, k=1, \ldots, 4$,

$$
\begin{aligned}
y_{1}(s)= & u_{1}(s)+\frac{3}{2 s+1} u_{3}(s)+\frac{5}{2 s+\frac{1}{5}} u_{4}(s) \\
& +f_{1}(s)+\frac{3}{2 s+1} f_{3}(s)+\frac{5}{2 s+1} f_{4}(s), \\
y_{2}(s)= & \frac{2}{s+1} u_{1}(s)+u_{2}(s) \\
& +\frac{2}{s+1} f_{1}(s)+f_{2}(s), \\
y_{3}(s)= & \frac{3}{5 s+1} u_{2}(s)+u_{3}(s) \\
& +\frac{3}{5 s+1} f_{2}(s)+f_{3}(s) .
\end{aligned}
$$


The set of residuals in the computational form is the following:

$$
\begin{aligned}
r_{1}(s)= & y_{1}(s)-u_{1}(s)-\frac{3}{2 s+1} u_{3}(s) \\
& -\frac{5}{2 s+1} u_{4}(s) \\
r_{2}(s)= & y_{2}(s)-\frac{2}{s+1} u_{1}(s)-u_{2}(s), \\
r_{3}(s)= & y_{3}(s)-\frac{3}{5 s+1} u_{2}(s)-u_{3}(s) .
\end{aligned}
$$

The internal form of residuals follows from Eqn. (9), which defines their dependence on faults:

$$
\begin{aligned}
& r_{1}(s)=f_{1}(s)+\frac{3}{2 s+1} f_{3}(s)+\frac{5}{2 s+1} f_{4}(s), \\
& r_{2}(s)=\frac{2}{s+1} f_{1}(s)+f_{2}(s), \\
& r_{3}(s)=\frac{3}{5 s+1} f_{2}(s)+f_{3}(s) .
\end{aligned}
$$

Thus

$$
\mathbf{G}^{F}(s)=\left[\begin{array}{cccc}
1 & 0 & \frac{3}{2 s+1} & \frac{5}{2 s+1} \\
\frac{2}{s+1} & 1 & 0 & 0 \\
0 & \frac{3}{5 s+1} & 1 & 0
\end{array}\right]
$$

Assume that changes in output signals do not appear, i.e., a system is in a steady state. Then residuals are in the form

$$
\begin{aligned}
& r_{1}(s)=f_{1}(s)+3 f_{3}(s)+5 f_{4}(s), \\
& r_{2}(s)=2 f_{1}(s)+f_{2}(s), \\
& r_{3}(s)=3 f_{2}(s)+f_{3}(s) .
\end{aligned}
$$

The column of Table 3 corresponding to a particular fault defines its signature. Directional vectors are as follows:

$$
\begin{array}{ll}
\mathbf{w}_{1}=[1,2,0], & \mathbf{w}_{2}=[0,1,3], \\
\mathbf{w}_{3}=[3,0,1], & \mathbf{w}_{4}=[5,0,0] .
\end{array}
$$

The sensitivity of residuals 35 on individual faults

Table 3. Directional signatures.

\begin{tabular}{|c|c|c|c|c|}
\hline & $f_{1}$ & $f_{2}$ & $f_{3}$ & $f_{4}$ \\
\hline \hline$r_{1}$ & 1 & 0 & 3 & 5 \\
\hline$r_{2}$ & 2 & 1 & 0 & 0 \\
\hline$r_{3}$ & 0 & 3 & 1 & 0 \\
\hline
\end{tabular}

$f_{k}$ can be considered qualitatively and represented by a binary diagnostic matrix. A pair of faults is distinguishable from all other single faults and other double faults when all columns of the binary diagnostic matrix are different. The binary diagnostic matrices for
Table 4. Binary diagnostic matrix for single faults.

\begin{tabular}{|c|c|c|c|c|}
\hline & $f_{1}$ & $f_{2}$ & $f_{3}$ & $f_{4}$ \\
\hline \hline$r_{1}$ & 1 & 0 & 1 & 1 \\
\hline$r_{2}$ & 1 & 1 & 0 & 0 \\
\hline$r_{3}$ & 0 & 1 & 1 & 0 \\
\hline
\end{tabular}

Table 5. Binary diagnostic matrix for double faults.

\begin{tabular}{|c|c|c|c|c|c|c|}
\hline & $f_{1} f_{2}$ & $f_{1} f_{3}$ & $f_{1} f_{4}$ & $f_{2} f_{3}$ & $f_{2} f_{4}$ & $f_{3} f_{4}$ \\
\hline \hline$r_{1}$ & 1 & 1 & 1 & 1 & 1 & 1 \\
\hline$r_{2}$ & 1 & 1 & 1 & 1 & 1 & 0 \\
\hline$r_{3}$ & 1 & 1 & 0 & 1 & 1 & 1 \\
\hline
\end{tabular}

single and double faults are presented in Tables 4 and 5 respectively.

Here " 1 " signifies that a single or a double fault of the column affects the residual of the row, while " 0 " means that it does not affect it. The elements in a double fault column are chosen as the "union" of the elements in the corresponding single fault columns. Each column is obtained in response to the single or double fault on top of the column. Since all the columns for single faults are different, the three residuals allow the distinction of all single faults. However, on the basis of structured residuals, we can clearly see that the double faults $f_{1}, f_{2}$ and $f_{1}, f_{3}$ and $f_{2}, f_{3}$ and $f_{2}, f_{4}$ are indistinguishable. Thus the residuals, in their row form, are not suitable for double fault isolation.

To facilitate double fault isolation, let us consider directional residuals. A set of perpendicular vectors for chosen pairs of faults is calculated as follows:

$$
\begin{aligned}
\mathbf{w}_{12} & =[1,2,0] \times[0,1,3]=[6,-3,1], \\
\mathbf{w}_{13} & =[1,2,0] \times[3,0,1]=[2,-1,-6], \\
\mathbf{w}_{23} & =[0,1,3] \times[3,0,1]=[1,9,-3], \\
\mathbf{w}_{24}=[0,1,3] \times[5,0,0] & =[0,15,-5] .
\end{aligned}
$$

Hence, the standard equations of planes in a three-dimensional parity space for the pairs of faults $f_{1}$ and $f_{2}, f_{1}$ and $f_{3}, f_{2}$ and $f_{3}$, and $f_{2}$ and $f_{4}$ are

$$
\begin{aligned}
& \mathbf{H}_{12}: 6 r_{1}-3 r_{2}+r_{3}=0, \\
& \mathbf{H}_{13}: 2 r_{1}-r_{2}-6 r_{3}=0, \\
& \mathbf{H}_{23}: r_{1}+9 r_{2}-3 r_{3}=0, \\
& \mathbf{H}_{24}: 15 r_{2}-5 r_{3}=0 .
\end{aligned}
$$

On the basis of the analysis of the current direction of the residuals vector we can isolate the appearing pairs of faults. For instance, let $\mathbf{r}=[1,1,-3]$. Then we can conclude the occurrence of double faults $f_{1}, f_{2}$, because the following coplanar condition is satisfied:

$$
\left(\mathbf{w}_{1} \times \mathbf{w}_{2}\right) \cdot \mathbf{r}=6 r_{1}-3 r_{2}+r_{3}=6-3-3=0 .
$$

The triple scalar product $\left(\mathbf{w}_{2} \times \mathbf{w}_{4}\right) \cdot \mathbf{w}_{2}=6$ for the pair of faults $f_{2}$ and $f_{4}$, and for fault $f_{2}$ is not equal zero. 
Therefore the fault $f_{2}$ is distinguishable from double faults $f_{2}, f_{4}$.

Two planes intersect on a line. Therefore, the pair of equations of planes taken together represents a line. For the distinguishable pair of faults $f_{1}$ and $f_{3}$ from the pair of faults $f_{2}$ and $f_{3}$ we take equations of $\mathbf{H}_{13}$ and $\mathbf{H}_{23}$.

Solving these equations, we get

$$
\left\{\begin{array} { l } 
{ 2 r _ { 1 } - r _ { 2 } - 6 r _ { 3 } = 0 } \\
{ r _ { 1 } + 9 r _ { 2 } - 3 r _ { 3 } = 0 }
\end{array} \Rightarrow \left\{\begin{array}{l}
r_{2}=0 \\
r_{1}=3 r_{3} .
\end{array}\right.\right.
$$

Hence, for a current residual vector in a steady state, which does not cover with $\left[3 r_{3}, 0, r_{3}\right]$, where $r_{3} \in \mathbb{R} \backslash\{0\}$, it can be concluded that double faults $f_{1}, f_{3}$ and $f_{2}, f_{3}$ are distinguishable. Moreover, the angles between the sample vector $\mathbf{r}=[0,1,-1]$ and the planes $\mathbf{H}_{13}$ and $\mathbf{H}_{23}$ are $\alpha_{13} \approx 33^{\circ}$ and $\alpha_{23} \approx 63^{\circ}$, respectively. Thus, the first plane is the closest to the actual residual vector $\mathbf{r}$ and the diagnosis about the occurrence of the double fault $f_{1}, f_{3}$ is more certain than that about the occurrence of the double fault $f_{2}, f_{3}$.

The robustness of double fault distinguishability can be achieved by designing secondary residuals. For determining the matrix $\mathbf{V}(s)$, we reduce the fault set to three elements $f_{1}, f_{2}, f_{3}$. With three primary residuals and three faults, the matrix

$$
\mathbf{G}^{F}(s)=\left[\begin{array}{ccc}
1 & 0 & \frac{3}{2 s+1} \\
\frac{2}{s+1} & 1 & 0 \\
0 & \frac{3}{5 s+1} & 1
\end{array}\right]
$$

is square and nonsingular, and so it has an inverse

$$
\left[\mathbf{G}^{F}(s)\right]^{-1}=\frac{1}{\boldsymbol{\Gamma}}\left[\begin{array}{ccc}
1 & A(s) & B(s) \\
C(s) & 1 & D(s) \\
E(s) & F(s) & 1
\end{array}\right],
$$

where

$$
\begin{aligned}
& A(s)=\frac{9}{(2 s+1)(5 s+1)}, \quad B(s)=\frac{-3}{2 s+1}, \\
& C(s)=\frac{-2}{s+1}, \\
& D(s)=\frac{6}{(s+1)(2 s+1)}, \\
& E(s)=\frac{6}{(s+1)(5 s+1)}, \quad F(s)=\frac{-3}{5 s+1},
\end{aligned}
$$

and

$$
\boldsymbol{\Gamma}=\operatorname{det}\left[\mathbf{G}^{F}(s)\right]=1+\frac{18}{(s+1)(2 s+1)(5 s+1)} .
$$

According to the requirement of the directions $\widetilde{\mathbf{w}}_{k}$ of faults $f_{1}, f_{2}, f_{3}$, the secondary residuals must be designed in such a way that all of the residual directions are perpendicular to each other. We can choose any two vectors $\widetilde{\mathbf{w}}_{1}$ and $\widetilde{\mathbf{w}}_{2}$, such that $\widetilde{\mathbf{w}}_{1} \perp \widetilde{\mathbf{w}}_{2}$, for example,

$$
\widetilde{\mathbf{w}}_{1}=[1,-2,0], \quad \widetilde{\mathbf{w}}_{2}=[6,3,1] .
$$

Then we find the third and last vector as follows:

$$
\widetilde{\mathbf{w}}_{3}=\left|\begin{array}{rrr}
\mathbf{i} & \mathbf{j} & \mathbf{k} \\
1 & -2 & 0 \\
6 & 3 & 1
\end{array}\right|=[-2,-1,15] .
$$

One can easily check that these three vectors create an orthogonal basis. Thus, the matrix $\mathbf{W}$ has the following form:

$$
\mathbf{W}=\left[\begin{array}{rrr}
1 & 6 & -2 \\
-2 & 3 & -1 \\
0 & 1 & 15
\end{array}\right]
$$

The next step in structured residual generation is the design of an appropriate matrix $\mathbf{D}(s)$ (22), which describes the dynamics of the response. It is important to underline the fact that the residual design property needs to be valid during fault transients as well, i.e., $\mathbf{D}_{k}$, for $k=1, \ldots, 4$ has to be the same for each element of the residual vector.

Let $\mathbf{D}(s)$ be the identity matrix I. Hence, the dynamics of all the secondary residuals are the same. The transformation matrix $\mathbf{V}$ becomes

$$
\mathbf{V}(s)=\mathbf{W}\left[\mathbf{G}^{F}(s)\right]^{-1} .
$$

Therefore, in order to enhance fault isolation, it is required to transform the primary residuals $\mathbf{r}$ by matrix $\mathbf{V}(s)$, according to 20). In a steady state, for the sample vector $\mathbf{r}=[0,1,3]$, which is the directional vector $\mathbf{w}_{2}$ of fault $f_{2}$, the form of secondary residuals is as follows:

$$
\begin{aligned}
& \mathbf{r}^{*}(s) \\
& \quad=\mathbf{W}\left[\mathbf{G}^{F}(s)\right]^{-1} \mathbf{r}(s) \\
& \quad=\frac{1}{19}\left[\begin{array}{rrr}
1 & 6 & -2 \\
-2 & 3 & -1 \\
0 & 1 & 15
\end{array}\right]\left[\begin{array}{rrr}
1 & 9 & -3 \\
-2 & 1 & 6 \\
6 & -3 & 1
\end{array}\right]\left[\begin{array}{l}
0 \\
1 \\
3
\end{array}\right] \\
& \quad=\frac{1}{19}\left[\begin{array}{c}
114 \\
57 \\
19
\end{array}\right]=\left[\begin{array}{l}
6 \\
3 \\
1
\end{array}\right]=\widetilde{\mathbf{w}}_{2} .
\end{aligned}
$$

Therefore, the occurrence of fault $f_{2}$ implies conversion of the residual vector $\mathbf{r}^{*}(s)=[6,3,1]$ to a pre-defined fault direction vector $\widetilde{\mathbf{w}}_{2}$. An analogical approach can be applied for designing secondary residuals for faults $f_{1}$ and $f_{3}$.

The pairs of double faults $f_{1}, f_{2}$ and $f_{1}, f_{3}$, and $f_{2}, f_{3}$ will occur on the three planes that are perpendicular to each other:

$$
\widetilde{\mathbf{H}}_{12} \perp \widetilde{\mathbf{H}}_{13} \perp \widetilde{\mathbf{H}}_{23},
$$


and this allows achieving the double fault distinguishability.

An analogical approach to designing secondary residuals can be applied to the distinguishability of other double faults, for instance, $f_{2}, f_{3}$ and $f_{2}, f_{4}$.

\section{Conclusions}

The assumption of the appearance of single faults allows a significant simplification of the algorithms of fault isolation, but is not always justified. The diagnosis of double faults turns out to be more complex than singular fault diagnosis. This is because the number of possible diagnoses increases in a significant way since double fault diagnoses are elements of the power set of single faults. In the case of realistic industrial installations the possibility of simultaneous occurrence of double faults must be taken into account.

The proposed method of double fault isolation is based on the investigation of the coplanarity of the current residual vector with the planes defined by the individual pairs of directional fault vectors. For steady state conditions, the hypothesis statement about the occurrence of double faults $f_{k}, f_{m}$ amounts to determining to which pre-defined double fault specific plane $\mathbf{H}_{k m}$ the observed residual vector is the closest. It should be noted that the directional residuals utilise the static dependence between a fault and a residual (i.e., a gain). Thus, this method does not include complete information about the binary relation fault-residuals (like knowledge about the dynamics of symptom forming).

When designing diagnostics systems, a crucial step is fault distinguishability. We proposed some hypotheses of double fault distinguishability conditions. The distinguishability for a given pair of faults from other faults and other pairs of faults is based on the use of knowledge about the existing fault specific directions. To facilitate double fault distinguishability, the residual set needs to have distinctive properties and unique characteristics of double faults. Thus, the residuals should be enhanced, that is, generated with specific isolation properties. The technique of the residual enhancement by the design of a secondary residual, which enables designers to improve double fault distinguishability with respect to diagnose conducted with the use of only primary residuals, was developed. An example of reasoning with the use of the proposed diagnostic methods was presented.

If diagnostic inference could be carried out taking into account double faults, knowledge of the dynamics of an effect of particular faults on the system outputs is necessary. In addition, it is assumed that this effect is linear and not integral in nature. Such data can be easily obtained for faults of measurement lines. Obtaining adequate transfer functions for faults of system elements and actuators requires the modelling of the system taking into account these faults, which is very difficult and sometimes outright impossible for many industrial systems. In the case where the system includes multiplicative faults, the validity of this approach is limited to a small neighbourhood of a linearization point. The method fails in the case of faults that change the object structure.

These assumptions are quite strong and limit the method applicability. However, if they are met, it is possible to achieve the distinguishability of double faults, which are not distinguished on the basis of binary signatures in a structured FDI approach and AI methods. There is no reason to use this method for pairs of double faults which are distinguished on the basis of binary signatures.

The cost of development of models with fault influence is much higher than in the case of input-output type models. Thus, the proposed method can be used for safety-critical systems, where design costs are negligible compared with losses in emergency situations.

\section{Acknowledgment}

This work was supported in part by the National Science Center under the project no. DEC-2011/01/B/ST7/06183.

\section{References}

Adam-Medina, M., Theilliol, D. and Sauter, D. (2003) Simultaneous fault diagnosis and robust model selection in multiple linear models framework, Proceedings of the 5th IFAC Symposium on Fault Detection, Supervision and $\mathrm{Sa}$ fety of Technical Processes, SAFEPROCESS, Washington, DC, USA, pp. 513-518.

Chen, J. and Patton, R.J. (1999). Robust Model Based Fault Diagnosis for Dynamic Systems, Kluwer Academic Publishers, Boston, MA.

Chen, R.H. and Speyer, J.L. (1999). Optimal stochastic multiple faults detection filter, Proceedings of the 38th IEEE Conference on Decision and Control, Phoenix, AL, USA, Vol. 5, pp. 4965-4970.

Clark, R.N. (1989). State estimation schemes for instrument fault detection, in R.J. Patton, P.M. Frank and R.N. Clark (Eds.), Fault Diagnosis in Dynamic Systems: Theory and Application, Prentice Hall, London.

Daigle, M., Koutsoukos, X. and Biswas, G. (2006). Multiple fault diagnosis in complex physical systems, 17th International Workshop on Principles of Diagnosis, Penaranda de Duero, Spain, pp. 69-76.

de Kleer, J. and Kurien, J. (2003). Fundamentals of model-based diagnosis, Proceedings of the 5th IFAC Symposium on Fault Detection, Supervision and Safety of Technical Processes, SAFEPROCESS 2003, Washington, DC, USA, pp. 25-36. 
de Kleer, J. and Williams, B.C. (1987). Diagnosing multiple faults, Artificial Intelligence 32(1): 97-130.

De-Persis, C. and Isidori, A. (2001). A geometric approach to nonlinear fault detection and isolation, IEEE Transactions on Automatic Control 46(6): 853-866.

Ding, S.X. (2008). Model-based Fault Diagnosis Techniques, Springer, Berlin/Heidelberg.

Frank, P.M. (1987). Fault diagnosis in dynamic systems via state estimations methods: A survey, in S.G. Tzafestas, M. Singh and G. Schmidt (Eds.), System Fault Diagnostics, Reliability and Related Knowledge-based Approaches, Vol. 2, D. Reidel Publishing Company, Dordrecht/Boston, MA/Lancaster/Tokyo.

Frank, P.M. (1991). Enhancement of robustness in observer-based fault detection, Proceedings of the IFAC/IMACS Symposium on Fault Detection, Supervision and Safety of Technical Processes, SAFEPROCESS, Baden-Baden, Germany, pp. 275-288.

Geltler, J. and Singer, D. (1990). A new structural framework for parity equation based failure detection and isolation, Automatica 26(2): 381-388.

Gertler, J. (1998). Fault Detection and Diagnosis in Engineering Systems, Marcel Dekker, Inc., New York, NY/Basel/Hong Kong.

Górny, B. (2001). Consistency-Based Reasoning in ModelBased Diagnosis, Ph.D. thesis, AGH University of Science and Technology, Cracow.

Hamscher, W., Console, L. and de Kleer, J. (1992). Readings in Model-Based Diagnosis, Morgan Kaufmann Publishers, San Mateo, CA.

Hashtrudi, S. and Massoumnia, M. (1999). Generic solvability of the failure detection and identification problem, Automatica 35(5): 887-893.

Hwee, T.N. (1991). Model-based, multiple-fault diagnosis of dynamic, continuous physical devices, IEEE Expert 6(6): $38-43$.

Isermann, R. (2006). Fault Diagnosis Systems. An Introduction from Fault Detection to Fault Tolerance, Springer-Verlag, New York, NY.

Khémiri, K., Ben Hmida, F., Ragot, J. and Gossa, M. (2011). Novel optimal recursive filter for state and fault estimation of linear stochastic systems with unknown disturbances, International Journal of Applied Mathematics and Computer Science 21(4): 629-637, DOI: 10.2478/v10006-011-0049-3.

Korbicz, J., Kościelny, J.M., Kowalczuk, Z. and Cholewa, W. (Eds.) (2004). Fault Diagnosis. Models, Artificial Intelligence, Applications, Springer, Berlin.

Kościelny, J.M. (1995). Fault isolation in industrial processes by dynamic table of states method, Automatica 31(5): 747-753.
Kościelny, J.M. (2001). Diagnostics of Automated Industrial Processes, Akademicka Oficyna Wydawnicza Exit, Warsaw, (in Polish).

Kościelny, J.M. and Łabęda, Z.M. (2007). Double fault distinguishability in linear systems, 8th Conference on Diagnostics of Processes and Systems, Stubice, Poland, pp. 45-52, (in Polish).

Kościelny, J.M., Bartyś, M. and Syfert, M. (2012). Method of multiple fault isolation in large scale systems, IEEE Transactions on Control Systems Technology 20(5): 1302-1310.

Ligęza, A. and Kościelny, J.M. (2008). A new approach to multiple fault diagnosis: A combination of diagnostic matrices, graphs, algebraic and rule-based models. The case of two-layer models, International Journal of Applied Mathematics and Computer Science 18(4): 465-476, DOI: 10.2478/v10006-008-0041-8.

Manders, E.J., Narasimhan, S., Biswas, G. and Mosterman, P. (2000). A combined qualitative/quantitative approach for fault isolation in continuous dynamic systems, 4th Symposium on Fault Detection, Supervision and Safety for Technical Processes, Budapest, Hungary, pp. 1074-1079.

Mattone, R. and de Luca, A. (2006). Relaxed fault detection and isolation: An application to a nonlinear case study, Automatica 42(1): 109-116.

Mosterman, P.J. and Biswas, G. (1999). Diagnosis of continuous valued systems in transient operating regions, IEEE Transactions on Systems, Man and Cybernetics, Part A 29(6): 554-565.

Patton, R.J., Frank, P.M. and Clark, R.N. (2000). Issues of Fault Diagnosis for Dynamic Systems, Springer, Berlin.

Sorsa, T. and Koivo, H.N. (1993). Application of artificial neural networks in process fault diagnosis, Automatica 29(4): 843-849.

Staroswiecki, M., Cassar, J.P. and Declerck, P. (2000). A structural framework for the design of FDI system in large scale industrial plants, in R.J. Patton, P.M. Frank and R.N. Clark (Eds.), Issues of Fault Diagnosis for Dynamic Systems, Springer-Verlag, Berlin.

Verde, C., Gentil, S. and Rosas, O. (2001). Fuzzy directional residuals evaluation for multileaks in pipelines, European Control Conference, Porto, Portugal, pp. 504-509.

Watanabe, K. and Hirota, S. (1991). Incipient diagnosis of multiple faults in chemical process via hierarchical artificial neural networks: Industrial electronics, control and instrumentation, IECON International Conference, Kobe, Japan, Vol. 2, pp. 1500-1505.

Watanabe, K. and Hou, L. (1992). An optimal neural network for diagnosing multiple faults in chemical processes. industrial electronics, control and instrumentation, Proceedings of the International Conference on Power Electronics and Motion Control, San Diego, CA, USA, Vol. 2, pp. 1068-1073. 


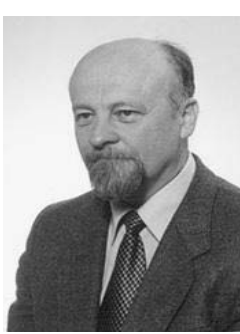

Jan Maciej Kościelny received his M.Sc. (in industrial automation), Ph.D. (in machine construction and operation) and D.Sc. (in automatic control and robotics) degrees as well as the professorial title from the Warsaw University of Technology in 1973, 1980, 1991 and 2003, respectively. He is the head of the Division of Automatic Control and Diagnosis of Industrial Processes at the Institute of Automatic Control and Robotics, Warsaw University of Technology. His research interests include the diagnostics of industrial processes and fault tolerant systems. He manages and heads research and development work leading to advanced systems of process monitoring and fault diagnosis along with their industrial applications. He is a member of the Committee on Automation and Robotics of the Polish Academy of Sciences, the Technical Committee: TC 6.4. Fault Detection, Supervision \& Safety of Technical Processes SAFEPROCESS of the IFAC, the editorial board of the International Journal of Applied Mathematics and Computer Science, and the Programme Committee of the Journal of Measurement Automation and Monitoring.

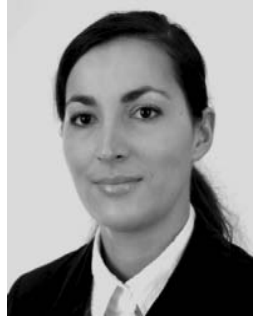

Zofia M. Labeda-Grudziak received the M.Sc. degree in mathematics from the Warsaw University of Technology, Faculty of Mathematics and Information Science, Poland, in 2006, and the $\mathrm{Ph} . \mathrm{D}$. degree in automatic control and robotics from the Warsaw University of Technology, Faculty of Mechatronics, in 2012. Currently, she is an assistant professor at the Institute of Automatic Control and Robotics, Warsaw University of Technology. Her research interests are in the general areas of fault diagnosis, industrial process modelling based on data mining techniques including both theory and applications, computational process modelling and simulation, and applied mathematics.

Received: 27 November 2011

Revised: 17 October 2012

Re-revised: 21 January 2013 